\title{
Spatial search and the Dirac equation
}

\author{
Andrew M. Childs $*$ and Jeffrey Goldstond \\ Center for Theoretical Physics, Massachusetts Institute of Technology, Cambridge, MA 02139, USA
}

(20 May 2004)

\begin{abstract}
We consider the problem of searching a $d$-dimensional lattice of $N$ sites for a single marked location. We present a Hamiltonian that solves this problem in time of order $\sqrt{N}$ for $d>2$ and of order $\sqrt{N} \log N$ in the critical dimension $d=2$. This improves upon the performance of our previous quantum walk search algorithm (which has a critical dimension of $d=4$ ), and matches the performance of a corresponding discrete-time quantum walk algorithm. The improvement uses a lattice version of the Dirac Hamiltonian, and thus requires the introduction of spin (or coin) degrees of freedom.
\end{abstract}

\section{INTRODUCTION}

Quantum mechanical computers can solve certain problems asymptotically faster than classical computers. One of the major advantages of quantum computation comes from a fast algorithm for the problem of finding a marked item among $N$ items. Whereas a classical computer requires $\Theta(N)$ steps to solve this problem, Grover showed that a quantum computer can solve it using only $O(\sqrt{N})$ steps [1], which is optimal [2].

To apply Grover's algorithm, it must be possible to quickly perform a reflection about a superposition of all possible items. However, this may not be feasible if the items are distributed in space and the algorithm is restricted to access them by local moves. For example, if the items are arranged on a one-dimensional line, simply traveling from one end of the line to the other requires $N$ moves, and a straightforward argument shows that no local algorithm, classical or quantum, can find a marked item in less time than $\Omega(N)$. But for other geometries, such as higher dimensional lattices, a quantum algorithm can conceivably achieve a speedup over the classical complexity of $\Theta(N)$.

Recently, there has been considerable progress in understanding the spatial search problem for quantum computers. Aaronson and Ambainis gave an algorithm that finds a marked item in the optimal time $O(\sqrt{N})$ for a lattice in $d>2$ dimensions, and in time $O\left(\sqrt{N} \log ^{2} N\right)$ for a two-dimensional lattice [3]. Their algorithm is based on a carefully optimized recursive search of subcubes, which raises the question of whether a simpler algorithm could solve the problem just as quickly (or perhaps even faster in two dimensions). In particular, it is interesting to consider quantum walk algorithms, which only use local, time-independent dynamics. Two distinct kinds of quantum walk algorithms have been considered. In the continuous-time quantum walk [4], the algorithm is described by a time-independent Hamiltonian connecting adjacent sites. In the discrete-time quantum walk $[5,6,7]$, the algorithm consists of repeated application

*amchilds@mit.edu

†oldston@mit.edu of a fixed local unitary transformation.

In [8], we considered a continuous-time quantum walk algorithm for the spatial search problem using no additional memory beyond the present location of the walker. We showed that this algorithm can find a single marked site in time $O(\sqrt{N})$ for dimensions $d>4$ and in time $O(\sqrt{N} \log N)$ in four dimensions. ${ }^{1}$ We also showed that this algorithm fails to provide an interesting speedup for dimensions $d<4$. Subsequently, Ambainis, Kempe, and Rivosh found a discrete-time quantum walk algorithm that works in lower dimensions 10]. This algorithm runs in time $O(\sqrt{N})$ for $d>2$ and in time $O(\sqrt{N} \log N)$ in two dimensions. Because a discrete-time quantum walk cannot be defined on a state space consisting only of the vertices of a graph [11], the algorithm of [10] necessarily uses additional memory (sometimes referred to as a "coin" in analogy to classical random walks). In this paper, we consider a continuous-time quantum walk using additional memory, and we show that it achieves the same running times as the discrete-time algorithm.

Through the analysis of 8], the failure of the continuous-time quantum walk algorithm for $d<4$ can be viewed as a consequence of a quadratic dispersion relation: states with small momentum have energy proportional to their momentum squared. If the dispersion were linear instead of quadratic, so that states with small momentum had energy proportional to their momentum, one might expect the algorithm to work whenever $d>2$. A natural way to achieve linear dispersion is to employ the massless Dirac equation. Indeed, using an appropriate lattice version of the Dirac Hamiltonian, we find a fast algorithm for spatial search in $d>2$. Because the Dirac particle necessarily possesses spin degrees of freedom, the resulting algorithm must have additional memory beyond the present location. Thus, although a continuous-time quantum walk can be defined without additional memory, we find that the additional degrees of freedom can

\footnotetext{
1 The run time $O(\sqrt{N} \log N)$ in $d=4$ can be achieved using amplitude amplification [9]. Using only classical repetition of the quantum walk, the algorithm requires $O\left(\sqrt{N} \log ^{3 / 2} N\right)$ steps. The same remark applies to the discrete-time quantum walk algorithm in $d=2$.
} 
improve the algorithm's performance.

\section{HAMILTONIANS FOR SPATIAL SEARCH}

In the Hamiltonian formulation of the spatial search problem, our goal is to write down a local Hamiltonian that will quickly transform a simple initial state, such as the uniform superposition over all lattice sites

$$
|s\rangle=\frac{1}{\sqrt{N}} \sum_{x}|x\rangle,
$$

to a state with substantial overlap on the marked state $|w\rangle$. In [8], we considered the Hamiltonian

$$
H_{[8]}=-\gamma L-|w\rangle\langle w| .
$$

Here the second term identifies the marked location, $\gamma$ is an adjustable parameter, and $L$ is the Laplacian of an $N$-site square lattice in $d$ dimensions, periodic in each direction with period $N^{1 / d}$. $L$ has matrix elements

$$
\left\langle x^{\prime}|L| x\right\rangle= \begin{cases}1 & x \text { adjacent to } x^{\prime} \\ -2 d & x=x^{\prime} \\ 0 & \text { otherwise }\end{cases}
$$

It is called the Laplacian because it is a discrete approximation to the continuum operator $\nabla^{2}$.

Since the free Hamiltonian $-\gamma L$ is translationally invariant, its eigenstates are the momentum eigenstates

$$
|k\rangle=\frac{1}{\sqrt{N}} \sum_{x} e^{i k \cdot x}|x\rangle
$$

where $k_{j}=\frac{2 \pi m_{j}}{N^{1 / d}}$, with $m_{j}=0, \pm 1, \ldots, \pm \frac{1}{2}\left(N^{1 / d}-1\right)$ for $N^{1 / d}$ odd, and $m_{j}=0, \pm 1, \ldots, \pm \frac{1}{2}\left(N^{1 / d}-2\right),+\frac{1}{2} N^{1 / d}$ for $N^{1 / d}$ even. The energy of the state $|k\rangle$ is $\mathcal{E}_{L}(k)=$ $2 \gamma\left(1-\sum_{j=1}^{d} \cos k_{j}\right)$. Thus, for small $|k|, \mathcal{E}_{L}(k) \approx \gamma k^{2}$. This quadratic dispersion relation ultimately gives rise to the critical dimension $d=4$ for the algorithm of 8 .

To achieve linear dispersion, we can replace $-\nabla^{2}$ by the massless Dirac Hamiltonian. In the general case of mass $m$, this Hamiltonian has the form 12

$$
H_{\text {Dirac }}=\sum_{j=1}^{d} \alpha_{j} p_{j}+\beta m
$$

where the operators $\alpha_{j}$ and $\beta$ act on spin degrees of freedom, and $p=-i \frac{\mathrm{d}}{\mathrm{d} x}$ is the momentum operator. If the spin operators $\alpha_{j}$ and $\beta$ satisfy the anticommutation relations

$$
\left\{\alpha_{j}, \alpha_{k}\right\}=2 \delta_{j, k}, \quad\left\{\alpha_{j}, \beta\right\}=0, \quad \beta^{2}=1,
$$

then one finds $H_{\text {Dirac }}^{2}=|p|^{2}+m^{2}$, as required for a relativistic particle. Thus for $m=0, H_{\text {Dirac }}$ has linear dispersion, $E_{\text {Dirac }}= \pm|p|$.
To write down the Dirac equation in $d$ dimensions, we need $d+1$ anticommuting operators. The minimal representation of the algebra (6) uses $2^{\lceil d / 2\rceil}$-dimensional matrices, and hence there are $2^{\lceil d / 2\rceil}$ spin components.

Now consider a lattice version of the massless Dirac Hamiltonian, equation (5) with $m=0$. The continuum operator $p_{j}$ can be discretely approximated as

$$
P_{j}|x\rangle=\frac{i}{2}\left(\left|x+e_{j}\right\rangle-\left|x-e_{j}\right\rangle\right),
$$

where $e_{j}$ is a unit vector in the $j$ direction. However, as we will see later, it turns out that simply taking the free Hamiltonian (5) using the lattice approximation (7) is insufficient. Instead, we will take ${ }^{2}$

$$
H_{0}=\omega \sum_{j} \alpha_{j} P_{j}+\gamma \beta L
$$

where both $\omega$ and $\gamma$ are adjustable parameters. For a Hamiltonian with spin degrees of freedom, translation invariance shows that the eigenstates have the form $|\eta, k\rangle$, where $|\eta\rangle$ is a (momentum-dependent) spin state. For (8), we find states with energies

$$
\mathcal{E}(k)= \pm \sqrt{\omega^{2} s^{2}(k)+\gamma^{2} c^{2}(k)},
$$

where

$$
s^{2}(k)=\sum_{j=1}^{d} \sin ^{2} k_{j}, \quad c(k)=2 \sum_{j=1}^{d}\left(1-\cos k_{j}\right) .
$$

For small momenta, we have $\mathcal{E}(k) \approx \pm \omega|k|$, which leads to a better search algorithm in low dimensions.

The full algorithm is as follows. We begin in the state $|\eta, s\rangle$, where $|\eta\rangle$ is any spin state and $|s\rangle$ is the uniform superposition (1). We then evolve with the Hamiltonian

$$
H=H_{0}-\beta|w\rangle\langle w|
$$

with parameters $\omega, \gamma$ to be determined in the analysis below, for a time $T$ also determined below. The goal is to choose the parameters $\omega$ and $\gamma$ so that for some $T$ as small as possible, the spatial component of the evolved state has a substantial overlap on $|w\rangle$.

\section{ANALYSIS OF THE ALGORITHM}

To analyze the algorithm, we would like to determine the spectrum of $H$ using our knowledge of the spectrum of $H_{0}$. We do this using the same techniques we applied

\footnotetext{
2 This choice is closely related to a standard remedy for the fermion doubling problem in lattice field theory [13, p. 27].
} 
to the Hamiltonian (2) in [8]. An eigenvector of $H$, denoted $\left|\psi_{a}\right\rangle$, with eigenvalue $E_{a}$, satisfies

$$
H\left|\psi_{a}\right\rangle=\left(H_{0}-\beta|w\rangle\langle w|\right)\left|\psi_{a}\right\rangle=E_{a}\left|\psi_{a}\right\rangle .
$$

Defining

$$
\left\langle w \mid \psi_{a}\right\rangle=\sqrt{R_{a}}\left|\phi_{a}\right\rangle
$$

where $\left|\phi_{a}\right\rangle$ is a normalized spin state, and $\sqrt{R_{a}}>0$ by choice of phases, we can rewrite (12) as

$$
\left(H_{0}-E_{a}\right)\left|\psi_{a}\right\rangle=\sqrt{R_{a}} \beta\left|\phi_{a}, w\right\rangle .
$$

Assuming $H_{0}-E_{a}$ is nonsingular, we can write the eigenstate of $H$ as

$$
\left|\psi_{a}\right\rangle=\frac{\sqrt{R}_{a}}{H_{0}-E_{a}} \beta\left|\phi_{a}, w\right\rangle .
$$

Consistency with (13) then gives the eigenvalue condition

$$
\left|\phi_{a}\right\rangle=F\left(E_{a}\right) \beta\left|\phi_{a}\right\rangle
$$

where

$$
F(E)=\left\langle w\left|\frac{1}{H_{0}-E}\right| w\right\rangle
$$

operates on the spin degree of freedom. To find eigenvalues of $H$, we must look for values of $E$ such that the spin operator $F(E) \beta$ has an eigenvector of eigenvalue 1 .

In addition to finding eigenvalues of $H$, we need some facts about its eigenvectors. The normalization condition on $\left|\psi_{a}\right\rangle$ gives

$$
\begin{aligned}
R_{a}^{-1} & =\left\langle\phi_{a}, w\left|\beta \frac{1}{\left(H_{0}-E_{a}\right)^{2}} \beta\right| \phi_{a}, w\right\rangle \\
& =\left\langle\phi_{a}\left|\beta F^{\prime}\left(E_{a}\right) \beta\right| \phi_{a}\right\rangle .
\end{aligned}
$$

We also need the overlap of $\left|\psi_{a}\right\rangle$ with eigenvectors of $H_{0}$. From (15) we have

$$
\left\langle\mathcal{E} \mid \psi_{a}\right\rangle=\frac{\sqrt{R_{a}}}{\mathcal{E}-E_{a}}\left\langle\mathcal{E}|\beta| \phi_{a}, w\right\rangle
$$

where $|\mathcal{E}\rangle$ is an eigenvector of $H_{0}$ with eigenvalue $\mathcal{E}$.

For the free Hamiltonian (요 , we find

$$
\begin{aligned}
F(E) \beta & =\left\langle w\left|\frac{H_{0}+E}{H_{0}^{2}-E^{2}}\right| w\right\rangle \beta \\
& =\frac{1}{N} \sum_{k} \frac{\gamma c(k)+\beta E}{\mathcal{E}(k)^{2}-E^{2}} \\
& =-\frac{\beta}{N E}+U(E)+\beta E V(E),
\end{aligned}
$$

where in (22) we have canceled terms that are odd in $k$, and

$$
\begin{aligned}
& U(E)=\frac{1}{N} \sum_{k \neq 0} \frac{\gamma c(k)}{\mathcal{E}(k)^{2}-E^{2}} \\
& V(E)=\frac{1}{N} \sum_{k \neq 0} \frac{1}{\mathcal{E}(k)^{2}-E^{2}} .
\end{aligned}
$$

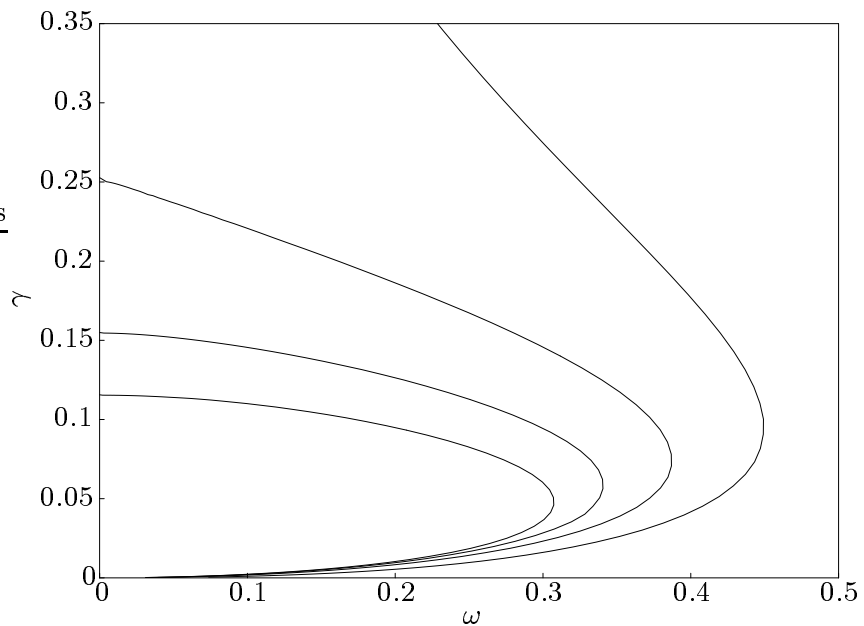

FIG. 1: Critical values of $(\omega, \gamma)$ for various dimensions. From rightmost curve to leftmost curve, $d=2,3,4,5$.

If $|E| \ll \mathcal{E}(k)$ for all $k \neq 0$, then we can Taylor expand $U(E)$ and $V(E)$ in powers of $E$. We will need only the leading order terms $U(0)$ and $V(0)$. For large $N$, we have

$$
U(0) \approx \frac{1}{(2 \pi)^{d}} \int_{-\pi}^{\pi} \frac{\gamma c(k) \mathrm{d}^{d} k}{\mathcal{E}(k)^{2}}
$$

which is a convergent integral regardless of $d$. For $d>2$ and $N$ large, we can also write $V(0)$ as a convergent integral,

$$
V(0) \approx \frac{1}{(2 \pi)^{d}} \int_{-\pi}^{\pi} \frac{\mathrm{d}^{d} k}{\mathcal{E}(k)^{2}} .
$$

In $d=2$, this integral is logarithmically infrared divergent, and instead we find

$$
V(0)=\frac{1}{4 \pi \omega^{2}} \ln N+O(1)
$$

Now suppose we choose $\omega$ and $\gamma$ such that $U(0)=1$. In this case, we are simply looking for a zero eigenvalue of $\beta\left(-\frac{1}{N E}+E V(0)\right)$. We find such eigenvalues with

$$
E_{ \pm} \approx \pm \frac{1}{\sqrt{V(0) N}}
$$

which indeed satisfy the condition $\left|E_{ \pm}\right| \ll \mathcal{E}(k)$ for all $k \neq 0$. These eigenvalues are degenerate in the spin space, i.e., any state $\left|\phi_{ \pm}\right\rangle$provides an eigenvector with the same eigenvalue.

The condition $U(0)=1$ can be satisfied by choosing $u(\omega / \gamma)=\gamma$, where $u(\omega / \gamma)=\gamma U(0)$ is a function only of $\omega / \gamma$. Figure 1 shows the critical curve in the $(\omega, \gamma)$ plane for $d=2$ through 5 . For any $\omega$ with $0<\omega<\omega^{*}$, where $\omega^{*}$ is some dimension-dependent threshold value, there are two values of $\gamma$ such that $U(0)=1$. Note that with $\omega=0$, we recover the results of [8]. Also, with 
$\gamma=0$, no solution of $U(0)=1$ exists, so it was essential to include this additional term (and the ability to fine tune its coefficient).

Having found the relevant eigenvalues, we need to determine the corresponding eigenstates. Using (19) we find

$$
R_{ \pm}^{-1} \approx \frac{1}{N E_{ \pm}^{2}}+V(0) \approx 2 V(0)
$$

and using (20) we find

$$
\left\langle\eta, s \mid \psi_{ \pm}\right\rangle=-\frac{\sqrt{R_{ \pm}}}{E_{ \pm} \sqrt{N}}\left\langle\eta|\beta| \phi_{ \pm}\right\rangle \approx \mp \frac{1}{\sqrt{2}},
$$

where we have chosen the eigenstate of $H$ with $\left|\phi_{ \pm}\right\rangle=$ $\beta|\eta\rangle$. Therefore we have

$$
|\eta, s\rangle \approx \frac{1}{\sqrt{2}}\left(\left|\psi_{-}\right\rangle-\left|\psi_{+}\right\rangle\right),
$$

and choosing $T=\pi /\left(2\left|E_{ \pm}\right|\right)$produces the state

$$
e^{-i H T}|\eta, s\rangle \approx \frac{1}{\sqrt{2}}\left(\left|\psi_{+}\right\rangle+\left|\psi_{-}\right\rangle\right)
$$

which has an overlap on $|\eta, w\rangle$ of $\sqrt{2 R_{ \pm}}$.

For $d>2$, we have shown that there is a $T=O(\sqrt{N})$ that gives a probability $O(1)$ of finding $w$. For $d=$ 2 , there is a $T=O(\sqrt{N \log N})$ that gives an amplitude $O(1 / \sqrt{\log N})$, so that amplitude amplification [9] can be used to find $w$ with a probability $O(1)$ in time $O(\sqrt{N} \log N)$.

\section{DISCUSSION}

We have described a continuous-time quantum walk algorithm for the spatial search problem. Using Dirac's insight of introducing spin to take the square root in a relativistic dispersion relation, we have found a Hamiltonian that locates a single marked item in the optimal time of $O(\sqrt{N})$ above the critical dimension $(d>2)$, and that runs in time $O(\sqrt{N} \log N)$ in $d=2$.

This algorithm is closely related to the discrete-time quantum walk search algorithm of [10]. Very similar techniques to the ones we have used in this paper can also be applied to discrete-time quantum walks [14]. This analysis for the algorithm of [10] closely parallels the analysis above, which highlights the similarity between the two kinds of algorithms. However, there are a few important differences. The continuous-time algorithm requires fine tuning the parameters $\omega$ and $\gamma$, whereas there is (apparently) no equivalent fine tuning in the discrete-time algorithm. Also, the discrete-time algorithm has noticeably different behavior depending on whether $N^{1 / d}$ is odd or even, a difference that is not seen in the continuous-time algorithm. In short, although the essential infrared features of the two kinds of algorithms are identical, their detailed behaviors differ.
In high dimensions, our algorithm is very wasteful in terms of the number of spin degrees of freedom: it uses a $2^{\lceil d / 2\rceil}$-dimensional spin space, whereas [8] shows that no spin degrees of freedom are required at all for $d>4$. In comparison, the discrete-time quantum walk search algorithm in [10] uses $2 d$ extra degrees of freedom. The Dirac particle in $d$ dimensions cannot be represented with fewer than $2^{\lceil d / 2\rceil}$ degrees of freedom, but a continuoustime search algorithm with only $d+1$ degrees of freedom can arise from reproducing the Dirac algebra (6) only on a subspace. If the operators $\alpha_{j}$ and $\beta$ satisfy

$$
\left\{\alpha_{j}, \alpha_{k}\right\}|\eta\rangle=2 \delta_{j, k}|\eta\rangle,\left\{\alpha_{j}, \beta\right\}|\eta\rangle=0, \beta|\eta\rangle=|\eta\rangle
$$

for some spin state $|\eta\rangle$, then the algorithm will work starting from the state $|\eta, s\rangle$. The condition (34) is sufficient to give $H_{0}^{2}|\eta, k\rangle=\mathcal{E}(k)^{2}|\eta, k\rangle$. The previous analysis then shows that

$$
\left|\psi_{a}\right\rangle=\frac{\sqrt{R_{a}}}{H_{0}-E_{a}}|\eta, w\rangle
$$

is an eigenstate of $H$ with eigenvalue $E_{a}$ provided $-\frac{1}{N E_{a}}+U\left(E_{a}\right)+E_{a} V\left(E_{a}\right)=1$, where $U(E)$ and $V(E)$ are as defined in equations (24) and (25). The rest of the analysis with two states $\left|\psi_{ \pm}\right\rangle$follows exactly as before. Finally, we see that (34) can be satisfied in a $(d+1)$-dimensional spin space with basis $|0\rangle,|1\rangle, \ldots,|d\rangle$, since in that case we can choose $\alpha_{j}=|0\rangle\langle j|+| j\rangle\langle 0|$, $\beta=2|0\rangle\langle 0|-I$, and $|\eta\rangle=|0\rangle$.

Unlike the algorithm of [8], the algorithm of this paper cannot be turned into an adiabatic algorithm. With the Hamiltonian (2), by starting in the state $|s\rangle$ and lowering the parameter $\gamma$ from a large value to zero sufficiently slowly, the adiabatic theorem guarantees that the system will remain near its ground state, ending up close to the state $|w\rangle$. In $d>4$, this can be done in time $O(\sqrt{N})$, and in $d=4$, it can be done in time $O\left(\sqrt{N} \log ^{3 / 2} N\right)$. However, in the algorithm of the present paper, states with $k=0$ are not the ground state of the free Hamiltonian (87); these states have zero energy, but this is in the middle of the spectrum. Although the adiabatic theorem applies to any eigenstate, not just the ground state, states near the middle of the spectrum of (11) with $\omega, \gamma$ small have very little overlap on $|w\rangle$, so that even perfectly adiabatic evolution produces a state far from the desired one.

Finally, we note that the actual complexity of the spatial search problem in $d=2$ is still an open question. A gap of $\log N$ remains between the best known algorithm and the lower bound of [2]. It would be interesting to improve the algorithm further or to show that no such improvement is possible.

\section{Acknowledgments}

We thank Andris Ambainis, Wim van Dam, Edward Farhi, Julia Kempe, and John Preskill for helpful discussions. AMC received support from the Fannie and 
John Hertz Foundation, and JG acknowledges the hospitality of the Caltech Institute for Quantum Information, where this work was supported in part by the National Science Foundation under grant EIA-0086038. This work was also supported in part by the Cambridge-MIT Insti- tute, by the Department of Energy under cooperative research agreement DE-FC02-94ER40818, and by the National Security Agency and Advanced Research and Development Activity under Army Research Office contract DAAD19-01-1-0656.
[1] L. K. Grover, Quantum mechanics helps in searching for a needle in a haystack, Phys. Rev. Lett. 79, 325 (1997), quant-ph/9706033.

[2] C. H. Bennett, E. Bernstein, G. Brassard, and U. Vazirani, Strengths and weaknesses of quantum computing, SIAM J. Comput. 26, 1510 (1997), quant-ph/9701001.

[3] S. Aaronson and A. Ambainis, Quantum search of spatial regions, in Proc. 44th IEEE Symposium on Foundations of Computer Science (2003), pp. 200-209, quant$\mathrm{ph} / 0303041$.

[4] E. Farhi and S. Gutmann, Quantum computation and decision trees, Phys. Rev. A 58, 915 (1998), quant$\mathrm{ph} / 9706062$.

[5] J. Watrous, Quantum simulations of classical random walks and undirected graph connectivity, J. Computer and System Sciences 62, 376 (2001), cs.CC/9812012.

[6] D. Aharonov, A. Ambainis, J. Kempe, and U. Vazirani, Quantum walks on graphs, in Proc. 33rd ACM Symposium on Theory of Computing (2001), pp. 50-59, quant$\mathrm{ph} / 0012090$.

[7] A. Ambainis, E. Bach, A. Nayak, A. Vishwanath, and J. Watrous, One-dimensional quantum walks, in Proc. 33rd ACM Symposium on Theory of Computing (2001), pp. $37-49$.

[8] A. M. Childs and J. Goldstone, Spatial search by quantum walk, quant-ph/0306054.

[9] G. Brassard, P. Høyer, M. Mosca, and A. Tapp, in Quantum Computation and Information, edited by S. J. Lomonaco and H. E. Brandt (AMS, Providence, RI, 2002), vol. 305 of AMS Contemporary Mathematics Series, quant-ph/0005055.

[10] A. Ambainis, J. Kempe, and A. Rivosh, Coins make quantum walks faster, quant-ph/0402107.

[11] D. A. Meyer, On the absence of homogeneous scalar unitary cellular automata, Phys. Lett. A 223, 337 (1996), quant-ph/9604011.

[12] P. A. M. Dirac, The quantum theory of the electron, Proc. Roy. Soc. London A 117, 610 (1928).

[13] M. Creutz, Quarks, Gluons, and Lattices (Cambridge University Press, Cambridge, 1983).

[14] A. M. Childs and J. M. Eisenberg, Quantum algorithms for subset finding, quant-ph/0311038. 